\title{
THE SOLUTION OF AN INTEGRAL EQUATION
}

\author{
C. NASIM
}

ABstract. Various methods are developed to solve the integral equation $f(x)=\int_{0}^{\infty} g(t) k(x t) d t$, when the Mellin transform $K(s)$ of the kernel function $k(x)$ is decomposable. Each method corresponds to the way $K(s)$ is decomposed: Namely (i) $K(s)=1 / L(1-s) M(1-s)$, (ii) $K(s)=H(s) / M(1-s)$ and (iii) $K(s)=N(s) H(s)$, where $L, M, N$ and $H$ are arbitrary functions of the complex variable $s$. Numerous special cases and examples are given to illustrate the technique and the advantage of these methods.

1. Introduction. Let us consider the integral equation

$$
g(x)=\int_{0}^{\infty} f(t) k(x t) d t, \quad x>0
$$

where the function $g(x)$ and $k(x)$ are known and $f(x)$ is to be found. Many techniques are known to solve this integral equation, depending upon the properties which the Mellin transform of the function $k(x)$, called the kernel, must satisfy. For example, the most familiar case is the classical one, where $k(x)$ is the Fourier kernel [2, Chapter III], that is, its Mellin transform $K(s)$, satisfies the following functional equation

$$
K(s) K(1-s)=1,
$$

where $s=c+i t,-\infty<t<\infty$, for some real $c$, and $k(x)$ in some sense is given by

$$
k(x)=\frac{1}{2 \pi i} \int_{c-i \infty}^{c+i \infty} K(s) \bar{x}^{s} d s .
$$

Then, by the Fourier inversion formula, the solution is

$$
(x)=\int_{0}^{\infty} k(x t) g(t) d t
$$

Received by the editors June 23, 1972 and, in revised form, October 16, 1972.

AMS (MOS) subject classifications (1970). Primary 45A05, 45E99; Secondary 44A05.

Key words and phrases. Fourier transform, Fourier kernel, Mellin transform, the Parseval Theorem, $L^{2}$-space, convergence in mean, Laplace and Stieltjes integral equations, Bessel functions. 
If $K(s)$ is a rational function involving gamma function, i.e.

$$
K(s)=\prod_{i=1}^{m} \Gamma\left(\alpha_{i} s+\beta_{i}\right) / \prod_{j=1}^{n} \Gamma\left(\alpha_{j} s+\beta_{j}\right),
$$

then by applying the operators $L$ and $L^{-1}$, to both sides of (1), $L$ being the Laplace operator, the solution can be found [1]. Also, the integral equation (1) is solvable in terms of Mellin transforms and the solution is,

$$
f(x)=\frac{1}{2 \pi i} \int_{c-i \infty}^{c+i \infty} \frac{G(1-s)}{K(1-s)} \bar{x}^{s} d s,
$$

where $G(s)$ is the Mellin transform of $g(x)$.

In this paper we shall give a method for solving the integral equation (1), when $K(s)$ is decomposable into arbitrary functions of $s$. The cases which we shall consider here are when $K(s)$ is decomposable into the following forms:

(i) $K(s)=1 / L(1-s) M(1-s)$,

(ii) $K(s)=H(s) / M(1-s)$,

(iii) $K(s)=N(s) H(s)$,

where $L, M, N$ and $H$ are arbitrary functions of $s ; s=c+i t,-\infty<t<\infty$.

2. Preliminaries. For simplicity, we shall confine ourselves to $L^{2}$-space and base the proofs given here upon the Mellin transforms. The following well-known theorems will be used and are found in [2, pp. 94, 95].

A. If $f(x) \in L^{2}(0, \infty)$, then

$$
F(s)=\int_{0}^{\infty} f(t) t^{s-1} d t,
$$

the integral converging to $F(s)$ in the mean square sense and $F(s) \in$ $L^{2}\left(\frac{1}{2}-i \infty, \frac{1}{2}+i \infty\right)$.

If $F(s) \in L^{2}\left(\frac{1}{2}-i \infty, \frac{1}{2}+i \infty\right)$, then

$$
f(x)=\frac{1}{2 \pi i} \int_{1 / 2-i \infty}^{1 / 2+i \infty} F(s) \bar{x}^{s} d s,
$$

the integral converging to $f(x)$ in the mean square sense and $f(x) \in L^{2}(0, \infty)$.

This defines $F(s)$ to be the Mellin transform of $f(x)$.

B. The Parseval Theorem. If $f(x)$ and $g(x)$ both belong to $L^{2}(0, \infty)$, and $F(s), G(s)$ are their respective Mellin transforms, then

$$
\int_{0}^{\infty} f(x) g(x) d x=\frac{1}{2 \pi i} \int_{1 / 2-i \infty}^{1 / 2+i \infty} F(s) G(1-s) d s .
$$


C. If $f(x)$ and $g(x)$ both belong to $L^{2}(0, \infty)$, then by the results (A) and (B), the integral equation (1) gives

$$
\frac{1}{2 \pi i} \int_{1 / 2-i \infty}^{1 / 2+i \infty} G(s) \bar{x}^{s} d s=\frac{1}{2 \pi i} \int_{1 / 2-i \infty}^{1 / 2+i \infty} F(1-s) K(s) \bar{x}^{s} d s .
$$

Therefore

and

$$
\frac{1}{2 \pi i} \int_{1 / 2-i \infty}^{1 / 2+i \infty}[G(s)-F(1-s) K(s)] \bar{x}^{s} d s=0,
$$

$$
G(s)=F(1-s) K(s)
$$

a.e. on $s=\frac{1}{2}+i t,-\infty<t<\infty$.

\section{Theorems.}

THEOREM 1. If $K(s)=1 / L(1-s) M(1-s), s=\frac{1}{2}+i t,-\infty<t<\infty$, then define $\Phi(s)$ as $\Phi(s)=L(s) G(1-s)$. Then the solution of the integral equation (1) is

$$
f(x)=\int_{0}^{\infty} \frac{1}{t} \varphi\left(\frac{1}{t}\right) m(x t) d t, \quad x>0,
$$

where $K(s), \Phi(s)$ and $M(s)$ are the respective Mellin transforms of the functions $k(x), \varphi(x)$ and $m(x)$, according to the result (A).

Proof. Since $\varphi(x) \in L^{2}(0, \infty)$, by hypothesis, therefore it is an easy matter to see that $(1 / x) \varphi(1 / x) \in L^{2}(0, \infty)$ also.

Now $m(x)$ and $(1 / x) \varphi(1 / x)$ both belong to $L^{2}(0, \infty)$, therefore the integral $\int_{0}^{\infty}(1 / t) \varphi(1 / t) m(x t) d t$, is absolutely convergent for $x>0$. By applying the Parseval Theorem (B) for Mellin transforms of $L^{2}$-functions, we obtain

$$
\int_{0}^{\infty} \frac{1}{t} \varphi\left(\frac{1}{t}\right) m(x t) d t=\frac{1}{2 \pi i} \int_{1 / 2-i \infty}^{1 / 2+i \infty} \Phi(s) M(s) \bar{x}^{s} d s .
$$

Now due to the result $(\mathrm{C})$ we have

$$
G(s)=F(1-s) K(s)=F(1-s) / L(1-s) M(1-s) .
$$

Therefore $F(s)=G(1-s) L(s) M(s)=\Phi(s) M(s)$. And

$$
\frac{1}{2 \pi i} \int_{1 / 2-i \infty}^{1 / 2+i \infty} \Phi(s) M(s) \bar{x}^{s} d s=\frac{1}{2 \pi i} \int_{1 / 2-i \infty}^{1 / 2+i \infty} F(s) \bar{x}^{s} d s=f(x),
$$

in the mean square sense by the result (A), as required.

THEOREM 2. If $K(s)=H(s) / M(1-s), s=\frac{1}{2}+i t,-\infty<t<\infty$, then define $\Phi(s)$ to be $\Phi(s)=G(1-s) / H(1-s)$. Then the solution of the integral equation 
(1) is

$$
f(x)=\int_{0}^{\infty} \frac{1}{t} \varphi\left(\frac{1}{t}\right) m(x t) d t, \quad x>0,
$$

where $K(s), \Phi(s)$ and $M(s)$ are as in Theorem 1.

Proof. Again $(1 / x) \varphi(1 / x)$ and $m(x)$ both belong to $L^{2}(0, \infty)$, therefore by the result $(B)$,

$$
\int_{0}^{\infty} \frac{1}{t} \Phi\left(\frac{1}{t}\right) m(x t) d t=\frac{1}{2 \pi i} \int_{1 / 2-i \infty}^{1 / 2+i \infty} \Phi(s) M(s) \bar{x}^{s} d s .
$$

Also, due to (C),

$$
G(s)=F(1-s) K(s)=F(1-s) H(s) / M(1-s),
$$

and therefore

$$
F(s)=G(1-s) M(s) / H(1-s)=\Phi(s) M(s) .
$$

Thus

$$
\frac{1}{2 \pi i} \int_{1 / 2-i \infty}^{1 / 2+i \infty} \Phi(s) M(s) \bar{x}^{s} d s=\frac{1}{2 \pi i} \int_{1 / 2-i \infty}^{1 / 2+i \infty} F(s) \bar{x}^{s} d s=f(x)
$$

in the mean square, by the result $(\mathrm{A})$, as required.

In the case when $K(s)=N(s) H(s)$, if we define $\Phi(s)=G(1-s) / H(1-s)$, then it is easy to see that the solution of the integral equation $\Phi(x)=$ $(1 / x) \int_{0}^{\infty} f(u) n(u / x) d u$ is also the solution of (1).

Note 1. In Theorem 1, if $L(s) M(s)=H(s)$, then we have the functional relation $K(s) H(1-s)=1$. The solution of (1) is then given by

$$
f(x)=\int_{0}^{\infty} g(t) h(x t) d t,
$$

which is the usual unsymmetrical Fourier integral formula [2, Chapter VIII]. Numerous examples of the relation between $h(x)$ and $k(x)$ can be found in the literature [2, Chapter VIII].

Note 2. In Theorem 1 , if $L(s) L(1-s)=1$, then $K(s)=L(s) / M(1-s)$ which is the case considered in Theorem 2.

Note 3. In Theorem 2, if $H(s) H(1-s)=M(s) M(1-s)$, then

$$
K(s) K(1-s)=1 \text {, }
$$

and then $k(x)$ is the Fourier kernel for the pair of functions $f(x)$ and $g(x)$. Hence the solution of (1) is

$$
f(x)=\int_{0}^{\infty} g(t) k(x t) d t .
$$


The results mentioned above will also hold in the function space $L(0, \infty)$. For this we need to impose appropriate conditions on the functions involved so that the results corresponding to (A) and (B) can be applied. These can be found in [2, pp. 46, 60].

4. Examples. The methods given above to solve the integral equation (1), essentially depend upon the decomposition of $K(s)$. This is done in such a way, so that the following integrals can be evaluated to give, respectively,

(i) $(1 / 2 \pi i) \int_{1 / 2-i \infty}^{1 / 2+i \infty} M(s) \bar{x}^{s} d s=m(x)$,

(ii) $(1 / 2 \pi i) \int_{1 / 2} \Phi(s) \bar{x}^{s} d s=\varphi(x)$, and

(iii) $\int_{0}^{\infty}(1 / t) \varphi(1 / t) m(x t) d t=f(x)$.

The advantage of these methods lies, therefore, in assuming that these above integrals involve elementary functions and are easy to evaluate or can simply be read from the tables ([3], [4]). To confirm this assumption, we give below a few examples. Throughout these examples the Mellin transforms are defined for the appropriate range of $\operatorname{Re}(s)$.

1. Laplace's integral equation.

$$
g(x)=\int_{0}^{\infty} f(t) e^{-x t} d t
$$

(i) Here $k(x)=\bar{e}^{x}$ and $K(s)=\Gamma(s)=\pi / \Gamma(1-s) \sin \pi s$, where $L(s)=$ $\sin \pi s$ and $M(s)=\Gamma(s)$. Thus $m(x)=\bar{e}^{x}$.

If $g(x)=\log x /(x-1)$, so that $G(s)=\pi^{2} \operatorname{cosec}^{2} \pi s$ [4, p. 538], then

$$
\Phi(s)=L(s) G(1-s)=\pi \operatorname{cosec} \pi s,
$$

whence $\varphi(x)=1 /(1+x)[3$, p. 308]. Thus by Theorem 1 ,

$$
f(x)=\int_{0}^{\infty} \frac{1}{t} \varphi\left(\frac{1}{t}\right) m(x t) d t=\int_{0}^{\infty} \frac{\bar{e}^{x t}}{1+t} d t=\Gamma(0, x) e^{x} \quad[3, \mathrm{p} .137] .
$$

2. Stieltjes's integral equation.

$$
\Psi(x)=\int_{0}^{\infty} \frac{f(u)}{x+u} d u
$$

By change of variable, we can write this as

$$
g(x)=\frac{1}{x} \Psi\left(\frac{1}{x}\right)=\int_{0}^{\infty} \frac{f(x)}{1+x t} d t .
$$

(i) Consider $k(x)=1 /(1+x)$. Then

$$
K(s)=\pi \operatorname{cosec} \pi s=\frac{\pi \Gamma(1-s)}{2 \Gamma(1-s) \sin \frac{1}{2} \pi s \cos \frac{1}{2} \pi s}
$$


where $H(s)=\Gamma(1-s) \operatorname{cosec} \frac{1}{2} \pi(1-s), \quad$ and $\quad M(s)=-(2 / \pi) \Gamma(s) \cos \frac{1}{2} \pi s$, thus $m(x)=-(2 / \pi) \cos x[3$, p. 348].

Now if $\Psi(x)=K_{0}\left(2 x^{1 / 2}\right)$, then $g(x)=\bar{x}^{1} K_{0}\left(2 x^{-1 / 2}\right)$, and $G(s)=\Gamma^{2}(1-s)$, and $\Phi(s)=G(1-s) / H(1-s)=\Gamma(s) \sin \frac{1}{2} \pi s$, whence $\varphi(x)=\sin x$.

Thus by Theorem 2

$$
f(x)=\int_{0}^{\infty} \frac{1}{t} \varphi\left(\frac{1}{t}\right) m(x t) d t=\frac{-2}{\pi} \int_{0}^{\infty} \frac{1}{t} \sin \left(\frac{1}{t}\right) \cos x t d t=-J_{0}\left(2 x^{1 / 2}\right)
$$

(ii) Now

$$
K(s)=\pi \operatorname{cosec} \pi s=\frac{\pi \operatorname{cosec} \pi s \Gamma(1-s)}{\Gamma(1-s)},
$$

where $H(s)=\pi \Gamma(1-s) \operatorname{cosec} \pi s$ and $M(s)=\Gamma(s)$. Thus $m(x)=\bar{e}^{x}$.

If $\Psi(s)=\log x /(x-1)$, then $g(x)=\log x /(x-1)$, and $G(s)=\pi^{2} \operatorname{cosec}^{2} \pi s$ $[4$, p. 538].

We define, $\Phi(s)=G(1-s) / H(1-s)=\Gamma(1-s)$, whence $\Phi(x)=\bar{x}^{1} \bar{e}^{1 / x}$. Thus by Theorem 2 ,

$$
f(x)=\int_{0}^{\infty} \frac{1}{t} \varphi\left(\frac{1}{t}\right) m(x t) d t=\int_{0}^{\infty} \bar{e}^{t(1+x)} d t=\frac{1}{1+x} .
$$

3. Cosine integral equation.

$$
g(x)=\int_{0}^{\infty} f(t) \cos x t d t .
$$

Here $k(x)=\cos x$, therefore $K(s)=\Gamma(s) \cos \frac{1}{2} \pi s=\Gamma(s) / \sec \frac{1}{2} \pi s$, where $H(s)=\Gamma(s)$ and $M(s)=\operatorname{cosec} \frac{1}{2} \pi s$. Thus $m(x)=1 /\left(1+x^{2}\right)$ [3, p. 345].

Now, if $g(x)=1 /(1+x)$, then $G(s)=\operatorname{cosec} \pi s$, and

$$
\Phi(s)=G(1-s) / H(1-s)=\Gamma(s),
$$

whence $\varphi(x)=\bar{e}^{x}$. Thus by Theorem 2 ,

$$
\begin{aligned}
f(x) & =\int_{0}^{\infty} \frac{1}{t} \varphi\left(\frac{1}{t}\right) m(x t) d t=\int_{0}^{\infty} \frac{u}{u^{2}+x^{2}} \bar{e}^{u} d n \\
& =c i(x) \cos x-s i(x) \sin x \quad[4, \text { p. 312] }
\end{aligned}
$$

4. Let $k(x)=J_{v}(u)$, and therefore

where

$$
K(s)=2^{s-1} \frac{\Gamma\left(\frac{1}{2} s+\frac{1}{2} v\right)}{\Gamma\left(\frac{1}{2} v-\frac{1}{2} s+1\right)}, \quad-R(v)<R(s)<\frac{3}{2},
$$

$$
H(s)=2^{s-1} \Gamma\left(\frac{1}{2} s+\frac{1}{2} v\right) \text { and } \quad M(s)=\Gamma\left(\frac{1}{2} v+\frac{1}{2} s+\frac{1}{2}\right),
$$


whence $m(x)=2 x^{v+1} \bar{e}^{x^{2}}$. Now if $g(x)=K_{v}(x)$, then $\Phi(s)=G(1-s) / H(1-s)$ gives $\varphi(x)=x^{v-1} e^{-1 / x^{2}}$. Thus

$$
\begin{aligned}
f(x)=\int_{0}^{\infty} \frac{1}{t} \varphi\left(\frac{1}{t}\right) m(x t) d t=2 x^{v+1} \int_{0}^{\infty} t \bar{e}^{t^{2}\left(1+x^{2}\right)} d t=\frac{x^{v+1}}{1+x^{2}}, & -1<R(v)<\frac{3}{2} .
\end{aligned}
$$

More generally if $g(x)=x^{\mu} K_{v-\mu}(x)$, then $f(x)=2^{\mu} \Gamma(\mu+1) x^{v+1} /\left(1+x^{2}\right)^{\mu+1}$

5. Let $k(x)=x^{1 / 2} H_{v}(x)$; then $[3$, p. 335] $K(s)=H(s) / M(1-s)$ where

$$
H(s)=\tan \frac{\pi}{2}\left(s+v+\frac{1}{2}\right) \text { and } M(s)=2^{s-1 / 2} \frac{\Gamma\left(\frac{1}{2} v+\frac{1}{2} s+\frac{1}{4}\right)}{\Gamma\left(\frac{1}{2} v-\frac{1}{2} s+\frac{3}{4}\right)}
$$

whence $m(x)=x^{1 / 2} J_{v}(x)$.

Now if $g(x)=x^{v-1 / 2} /\left(1+x^{2}\right)$, then $\Phi(s)=G(1-s) / H(1-s)$ gives $\varphi(x)=$ $x^{1 / 2-v} /\left(1+x^{2}\right)$. Thus by Theorem 1 ,

$$
\begin{array}{r}
f(x)=\int_{0}^{\infty} \frac{1}{t} \varphi\left(\frac{1}{t}\right) m(x t) d t=x^{1 / 2} \int_{0}^{\infty} \frac{t^{v+1}}{1+t^{2}} J_{v}(x t) d t=x^{1 / 2} K_{v}(x), \\
-1<R(v)<\frac{3}{2} \quad[4, \text { p. 686]. }
\end{array}
$$

ACKNOWLEDGEMENT. The preparation of this paper was assisted by a grant from the National Research Council of Canada.

\section{REFERENCES}

1. C. Fox, Solving integral equations by $L$ and $L^{-1}$ operators, Proc. Amer. Math. Soc. 34 (1972), 299-306.

2. E. C. Titchmarsh, Theory of Fourier integral, Clarendon Press, Oxford, 1937.

3. A: Erdélyi et al., Tables of integral transforms. Vol. 1, McGraw-Hill, New York, 1954. MR 15, 868.

4. I. S. Gradšteinn and I. M. Ryžik, Tables of integrals, series and products, Fizmatgiz, Moscow, 1963; English transl., Academic Press, New York, 1965. MR 28 \#5198; MR 33 \#5952.

Department of Mathematics, University of Calgary, Calgary, Alberta, Canada 